\title{
Influência de uma sessão de exercício aeróbico submáximo e máximo sobre o transporte mucociliar e função autonômica em tabagistas
}

\author{
Influence of a submaximal and maximal aerobic exercise session on mucociliary clearance and \\ autonomic function in smokers
}

Influencia de una sesión de ejercicio aeróbico submáximo y máximo sobre el transporte mucociliar y la función autonómica en fumadores

Iara Buriola Trevisan', Luiz Carlos Marques Vanderlei², Renata Marques David ${ }^{3}$, Caroline Pereira Santos ${ }^{4}$, Ercy Mara Cipulo Ramos ${ }^{5}$, Dionei Ramos 6

\begin{abstract}
RESUMO I O objetivo do estudo foi avaliar e correlacionar o comportamento da depuração mucociliar e do sistema nervoso autônomo de fumantes após sessões de exercício aeróbico submáximo e máximo. Foram avaliados 25 fumantes e 15 não fumantes, entre 30 e 50 anos. Ambos os grupos foram submetidos ao teste do tempo de trânsito de sacarina (TTS) e variabilidade da frequência cardíaca (VFC) antes e após uma sessão de exercício submáximo (teste de caminhada de seis minutos) e máximo (teste de exercício cardiopulmonar). Teste t pareado ou Wilcoxon foi utilizado para análise intragrupos e o teste t não pareado ou Mann-Whitney para a análise intergrupos. A correlação foi realizada utilizando os coeficientes de Pearson ou Spearman ( $p<0,05)$. Houve redução significativa do TTS após exercícios submáximo e máximo em ambos os grupos. Após o exercício submáximo, ambos grupos apresentaram redução significativa do intervalo $\mathrm{RR}$ e aumento da FC em comparação ao repouso, no grupo
\end{abstract}

de não fumantes houve reduções significativas nos índices RMSSD, HFms² e SD1. Após o exercício máximo, ambos grupos apresentaram reduções significativas no SDNN, RMSSD, intervalo RR, LF e HF, em $\mathrm{ms}^{2}$ e un, SD1 e SD2, além do aumento da FC, LFun e da razão LF/HF. Houve correlação positiva entre TTS e LFms ${ }^{2}(r=0,520$, $p=0,008)$ após o exercício máximo para o grupo de fumantes. Conclui-se que independentemente da intensidade do exercício aeróbio, houve um aumento na depuração mucociliar em fumantes, mas essa alteração parece ser influenciada pelo sistema nervoso autônomo apenas frente o exercício máximo.

Descritores | Depuração Mucociliar; Sistema Nervoso Autônomo; Tabagismo; Exercício.

ABSTRACT I The aim of this study was to evaluate and to correlate the behavior of mucociliary clearance and the autonomic nervous system of smokers after submaximal and

Estudo realizado no Departamento de Fisioterapia, Faculdade de Tecnologia e Ciência, Universidade Estadual Paulista (Unesp), Presidente Prudente (SP), Brasil.

Este trabalho é parte integrante da dissertação Transporte mucociliar e função autonômica de tabagistas submetidos ao esforço físico submáximo e máximo.

Trabalho apresentado no European Respiratory Society International Congress, 26 a 30 de setembro de 2015, Amsterdam. 'Universidade Estadual Paulista Júlio de Mesquita Filho (Unesp) - Presidente Prudente (SP) - Brasil. E-mail: iara_buriola@hotmail.com. Orcid: 0000-0003-0743-3231

${ }^{2}$ Universidade Estadual Paulista Júlio de Mesquita Filho (Unesp) - Presidente Prudente (SP) - Brasil. E-mail: Icm.vanderlei@unesp.br. Orcid: 0000-0002-1891-3153

3 Universidade Estadual Paulista (Unesp) - Presidente Prudente (SP), Brasil. E-mail: renatamdavid0@gmail.com. Orcid: 0000-0002-2240-6832 4Universidade Estadual Paulista Júlio de Mesquita Filho (Unesp) - Presidente Prudente (SP) - Brasil. E-mail: carolinepereirasantos@yahoo. com.br. Orcid: 0000-0001-7608-9986

${ }_{5}^{5}$ niversidade Estadual Paulista Júlio de Mesquita Filho (Unesp) - Presidente Prudente (SP) - Brasil. E-mail: ercy@bol.com.br. Orcid: 0000-0002-3310-7336

${ }^{6}$ Universidade Estadual Paulista Júlio de Mesquita Filho (Unesp) - Presidente Prudente (SP) - Brasil. E-mail: dionei-ramos@bol.com.br. Orcid: 0000-0002-2956-7399 
maximal aerobic exercise sessions. We evaluated 25 smokers and 15 nonsmokers aged between 30 and 50 years. Both groups were submitted to the saccharin transit time (STT) test and heart rate variability (HRV) before and after a submaximal (six-minute walk test) and maximal (cardiopulmonary test) exercise. Paired t-test or Wilcoxon were used for intragroup analysis and the unpaired t-test or Mann-Whitney for intergroup analysis. The correlation was performed using Pearson or Spearman coefficients $(p<0.05)$. Saccharine transit time reduced significantly after submaximal and maximal exercises in both groups. After the submaximal exercise, both groups presented significant reduction of the RR interval and increased heart rate (HR). In the nonsmoker group there were significant reductions in the RMSSD, HFms ${ }^{2}$ and SD1 indexes. After maximal exercise, both groups showed significant reductions in SDNN, RMSSD, RR, LF and HF interval, in $\mathrm{ms}^{2}$ and normalized units, SD1 and SD2, in addition to the increase in HR, LFun, and LF/HF ratio. STT positively correlated with LFms ${ }^{2}(r=$ 0.520, $p=0.008$ ) after the maximal exercise for the smoker group. We concluded, that regardless of the intensity of aerobic exercise, mucociliary clearance increases in smokers, but this alteration seems to be influenced by the autonomic nervous system only during maximum exercise.

Keywords | Mucociliary Clearance; Autonomic Nervous System; Smoking; Exercise.

RESUMEN | El objetivo de este estudio fue evaluar y correlacionar el comportamiento de la depuración mucociliar y del sistema nervioso autónomo de fumadores después de sesiones de ejercicio aeróbico submáximo y máximo. Se evaluaron a 25 fumadores y a 15 no fumadores de entre 30 y 50 años de edad. Ambos grupos se sometieron a la prueba de tiempo de tránsito de sacarina (TTS) y la variabilidad de la frecuencia cardíaca (VFC) antes y después de una sesión de ejercicio submáximo (prueba de caminata de seis minutos) y de ejercicio máximo (prueba de esfuerzo cardiopulmonar). Para el análisis intragrupo se utilizó la prueba t pareada o Wilcoxon, y para el análisis intergrupal, la prueba t no pareada o Mann-Whitney. Para realizar la correlación se utilizaron los coeficientes de Pearson o Spearman $(p<0,05)$. Hubo una reducción significativa en TTS después de ejercicios submáximo y máximo en ambos grupos. Después del ejercicio submáximo, ambos grupos mostraron una reducción significativa en el intervalo RR y un aumento en la FC en comparación con el reposo; en el grupo de no fumadores hubo reducciones significativas en los índices RMSSD, HFms ${ }^{2}$ y SD1. Después del ejercicio máximo, ambos grupos mostraron reducciones significativas en SDNN, RMSSD, intervalo RR, LF y HF, en $\mathrm{ms}^{2}$ y un, SD1 y SD2, además de un aumento de FC, LFun y la relación LF/HF. Hubo una correlación positiva entre TTS y LFms ${ }^{2}(r=0,520$, $p=0,008$ ) después del ejercicio máximo para el grupo de fumadores. Se concluye que, de manera independiente a la intensidad del ejercicio aeróbico, hubo un aumento de la depuración mucociliar en los fumadores, pero este cambio parece haber sido influido por el sistema nervioso autónomo solamente en el ejercicio máximo. Palabras clave | Depuración Mucociliar; Sistema Nervioso Autónomo; Tabaquismo; Ejercicio.

\section{INTRODUÇÃO}

O tabagismo pode promover diversas alterações no organismo, dentre elas, as que ocorrem na modulação autonômica ${ }^{1}$ e no transporte mucociliar (TMC) $)^{2,3}$. Na modulação autonômica, ocorre ativação simpática e retirada vagal ${ }^{4}$ e em decorrência das alterações da mucosa respiratória, composição do muco, estrutura e função ciliar ${ }^{2,5,6}$, tabagistas apresentam aumento do tempo de TMC o que pode estar diretamente associado a carga tabagística ${ }^{6}$, entretanto, após a cessação do tabagismo esse quadro pode ser revertido ${ }^{7}$.

O exercício físico também pode acelerar o tempo da $\mathrm{TMC}^{8}$, sugerindo que a hiperventilação pulmonar e estimulação simpática ${ }^{9}$ após o exercício acelera o batimento ciliar, o que facilita a eliminação de partículas nocivas ao sistema respiratório, potencializando sua ação de defesa ${ }^{10,11}$. No entanto, em tabagistas ainda não

está claro os efeitos do exercício aeróbico submáximo e máximo na depuração mucociliar e a influência do sistema nervoso autônomo frente a diferentes intensidades de exercício.

Portanto, considerando que o tabagismo promove alterações tanto no TMC quanto na modulação autonômica e que o exercício físico pode acelerar o TMC o que, pelo menos em partes, está relacionado à alterações do sistema nervoso autônomo, é de suma importância para a prática clínica avaliar se isso também ocorre em indivíduos tabagistas frente à diferentes intensidades do exercício físico, já que o exercício físico é um recurso terapêutico que pode ser utilizado no tratamento clínico de tabagistas. Portanto, este estudo teve por objetivo avaliar as alterações e relações do comportamento do TMC nasal e da modulação autonômica de tabagistas após um estímulo de exercício aeróbico submáximo e máximo. 


\section{METODOLOGIA}

Estudo prospectivo e transversal realizado com 69 participantes com idade entre 30 e 50 anos, alocados para o grupo tabagistas $(n=44)$ e grupo de não tabagistas (controle, $n=25$ ). Destes, 29 perderam follow-up, sendo 14 por não comparecimento e 15 por outras razões. Assim, a amostra final inclui 40 participantes, sendo 25 tabagistas e 15 não tabagistas (Figura 1).

Os critérios de inclusão foram: indivíduos fisicamente inativos (que não realizassem atividade física regular por 20 minutos contínuos, três vezes na semana), função pulmonar normal confirmada por espirometria e ausência de doenças cardiorrespiratórias, metabólicas, neuromusculares e esqueléticas conhecidas. Para o grupo tabagista foram incluídos indivíduos com histórico de consumo tabagístico de, no mínimo, 20 cigarros por dia por pelo menos um ano. Os critérios de exclusão foram: não compreensão ou não colaboração do voluntário em relação aos procedimentos e métodos da pesquisa, ausência em um dos protocolos de exercício, infecções respiratórias recentes, desvio de septo nasal, histórico de cirurgia ou trauma nasal e etilismo ou qualquer condição adversa de saúde que pudesse interferir no desempenho do exercício ou modulação autonômica.

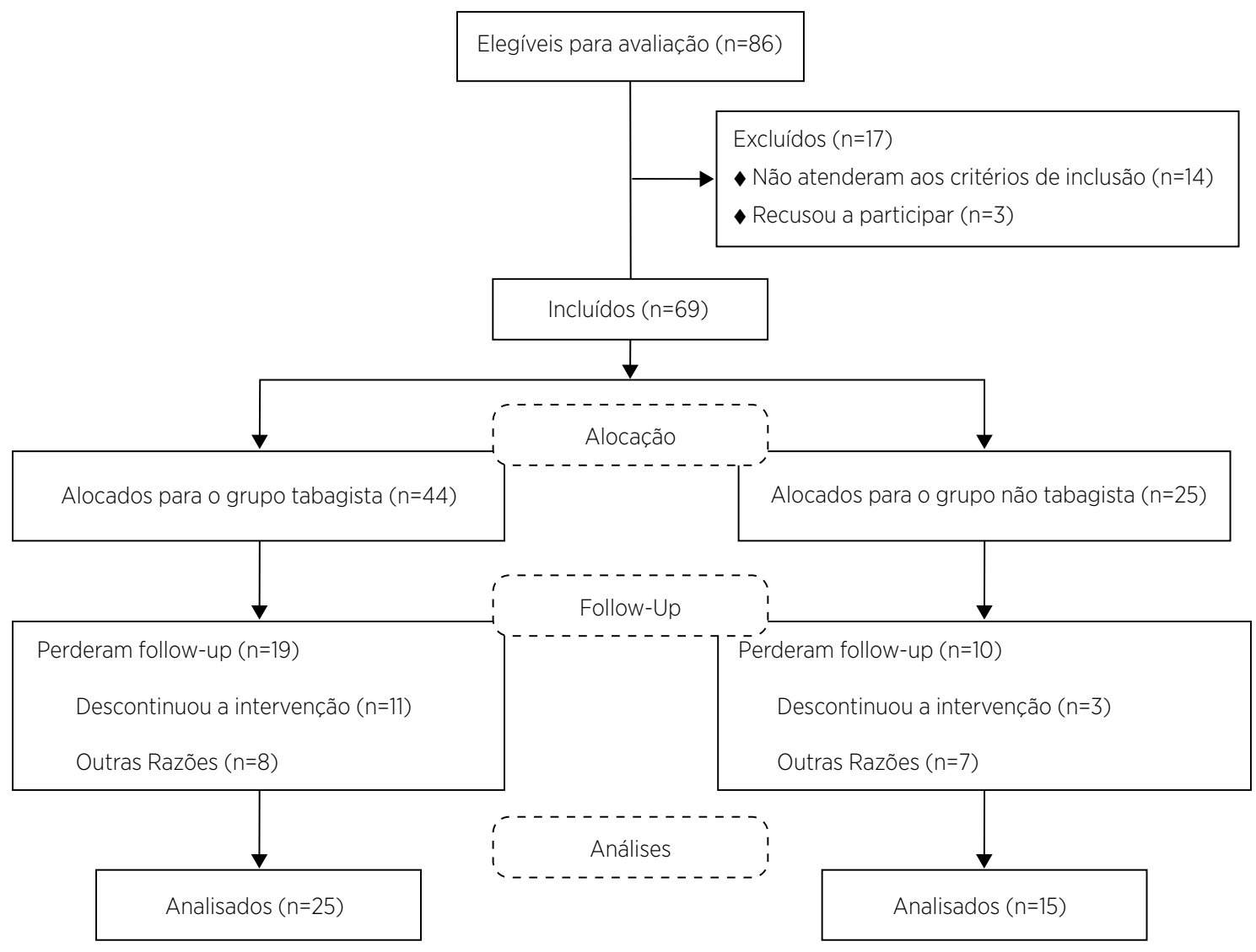

Figura 1. Fluxograma do estudo

Este estudo foi aprovado pelo Comitê de ética em Pesquisa (processo no 223.033). Os sujeitos foram informados sobre os objetivos e procedimentos do estudo e após assinarem o termo de consentimento foram incluídos na pesquisa.

O protocolo foi realizado em três dias não consecutivos. Todas as avaliações foram realizadas no período matutino em ambiente com temperatura $\left(22,93 \pm 1,32^{\circ} \mathrm{C}\right)$ e umidade relativa do ar controladas $(53,96 \pm 3,82 \%)$. Os indivíduos foram instruídos a realizarem refeições leves $2-\mathrm{h}$ antes das avaliações e a se absterem de álcool, cafeína, cigarro e atividade física vigorosa por $12-\mathrm{h}$ antes das avaliações.

No primeiro dia de protocolo, todos os indivíduos foram submetidos a uma avaliação inicial, que incluía entrevista para coleta de dados pessoais, mensuração antropométrica, estado geral de saúde (comorbidades e história de cirurgia e trauma nasal), histórico tabagístico e nível de dependência à nicotina avaliado por meio do teste de Fagerstrom $^{12}$. A mensuração do monóxido de carbono do ar exalado (COex, ponto de corte de 
$10 \mathrm{ppm}^{13}$ ) foi avaliado por meio da técnica padrão usando o analisador de monóxido de carbono (Micro CO, Micro Medical Ltd. apparatus, Rochester, Kent, UK) $)^{14}$, e a avaliação da função pulmonar foi realizada por meio da espirometria (VEF1/CVF >70\% - Spirobank 3.6, Medical International Research, Rome, Italy) de acordo com as normas do American Thoracic Society e European Respiratory Society ${ }^{15}$ utilizando valores de referência para a população brasileira ${ }^{16}$.

Os outros dois dias de protocolo foram realizados com um intervalo mínimo de 48 horas entre eles. Em um dos dias realizou-se o exercício físico submáximo por meio do teste de caminhada de seis minutos (TC6') de acordo com o guia da American Thoracic Society ${ }^{17}$ e no outro o exercício físico máximo por meio do teste progressivo exaustivo em esteira ergométrica ${ }^{18} \mathrm{com}$ analisador de gases (VO2000, Medical Graphics, EUA). Foi considerado exercício máximo quando pelo menos dois dos seguintes critérios foram atendidos: FC atingida $>90 \%$ da FC máxima (220-idade); percepção subjetiva de esforço ${ }^{19}$ acima de 17; eventual platô no gráfico de consumo de oxigênio (VO2 1/min) diante de um aumento na carga de esforço e relação dos equivalentes respiratórios dióxido de carbono e consumo de oxigênio $(\mathrm{QR})>1,1^{19-21}$.

Antes da realização dos protocolos de exercício físico, os indivíduos permaneceram sentados em repouso por 20 minutos para estabilização das variáveis cardiorrespiratórias e captação da frequência cardíaca batimento a batimento por meio de um cardiofrequencímetro Polar S810i (Polar Electro, Kempele, Finlândia) $)^{22,23}$. Em seguida, realizou-se a coleta de COex e foi realizado o teste de tempo de trânsito de sacarina (TTS) 2,3,7,8,24,25.

Os protocolos de exercícios foram iniciados após a primeira percepção de um gosto doce na boca. Para evitar a influência da frequência respiratória sobre o TMC as avaliações foram repetidas após dez minutos do término de ambos os exercícios, deste modo, coletou-se novamente o COex, em seguida o TTS e a frequência cardíaca batimento a batimento foi coletada até a percepção do gosto da sacarina.

A frequência cardíaca batimento a batimento foi registrada em repouso, na posição sentada durante 20 minutos antes e após os protocolos de exercício até a percepção do gosto doce na boca durante o TTS. Para análise dos índices de variabilidade da frequência cardíaca (VFC) em cada protocolo, foram selecionados 256 intervalos RR (oscilações dos intervalos entre batimentos cardíacos consecutivos) na fase mais estável durante o repouso inicial e durante a avaliação final do TTS, ou seja, 256 batimentos cardíacos prévios ao momento exato que os indivíduos relataram terem sentido o gosto da sacarina.

O trecho selecionado foi submetido a uma filtragem digital (software Polar Precision Performance SW, versão 4.01.029) complementada por manual e somente séries com mais de $95 \%$ de batimentos sinusais foram incluídas no estudo. O software Kubios (Biosignal and Medical Image Analysis Group, Department of Physics, University of Kuopio, Kuopio, Finland) ${ }^{26}$ foi utilizado para cálculo dos índices de VFC, como: intervalos RR, raiz quadrada da média do quadrado das diferenças entre os intervalos $\mathrm{RR}$ normais adjacentes (RMSSD, expresso em ms) e desvio-padrão da média dos intervalos RR normais (SDNN, expresso em $\mathrm{ms}$ ). No domínio frequência foram analisados os componentes espectrais de baixa (LF, 0,04-015 Hz) e alta frequência ( $\mathrm{HF}, 0,15-0,40 \mathrm{~Hz}$ ), em unidades normalizadas (un) e em milissegundos ao quadrado (ms2) e a razão entre estes componentes (relação LF/HF) ${ }^{22,27,28}$. A análise realizada no domínio da frequência foi calculada utilizando o algoritmo da Transformada Rápida de Fourier ${ }^{29}$.

O plot de Poincaré foi realizado para calcular os seguintes índices: SD1 (desvio-padrão da variabilidade instantânea batimento a batimento) e SD2 desvio-padrão dos intervalos $\mathrm{RR}$ contínuos da variabilidade de longa duração) $)^{22,27,29}$.

Para análise foi utilizado o programa estatístico SPSS 22.0. A normalidade dos dados foi realizada por meio do teste de Shapiro-Wilk. Para a comparação intragrupos foi utilizado o teste t pareado ou teste de Wilcoxon, de acordo com a normalidade dos dados. Para análise entre os grupos utilizou-se o teste $t$ não pareado para dados com distribuição normal ou teste de MannWhitney para aqueles com distribuição não normal. As análises de correlações foram realizadas por meio dos coeficientes de Pearson ou Spearman de acordo com a normalidade dos dados. O nível de significância adotado para todos os testes foi de $\mathrm{p}<0,05$.

\section{RESULTADOS}

$\mathrm{Na}$ Tabela 1 estão apresentadas as características basais dos dois grupos avaliados. 
Tabela 1. Caracterização da amostra quanto ao gênero, idade, medidas antropométricas, índices espirométricos, capacidade física e histórico tabagístico

\begin{tabular}{|c|c|c|c|}
\hline Variáveis & Grupo Tabagista $(n=25)$ & Grupo Controle $(n=15)$ & $p$ \\
\hline Gênero (F/M) & $11 / 14$ & $9 / 6$ & $0,327 \delta$ \\
\hline Idade (anos) & $43,0(35,0-46,0)$ & $46,0(32,0-49,0)$ & $0,211 \ddagger$ \\
\hline IMC $\left(\mathrm{kg} / \mathrm{m}^{2}\right)$ & $27,0(24,2-30,7)$ & $26,2(22,7-29,9)$ & $0,419^{+}$ \\
\hline $\mathrm{VEF}_{1} / \mathrm{CVF}$ (\% predito) & $85,8(82,2-90,5)$ & $85,0(82,0-86,0)$ & $0,679 \ddagger$ \\
\hline CVF(\% predito) & $97,0(87,5-107,5)$ & $92,0(90,0-107,0)$ & $0,638^{+}$ \\
\hline $\mathrm{VEF}_{1}(\%$ predito $)$ & $93,0(81,5-104,0)$ & $97,0(91,0-108,0)$ & $0,206^{+}$ \\
\hline DTC6 (\%) & $92,0(85,0-100,0)$ & $102,8(100,5-109,5)$ & $<0,0001 t^{*}$ \\
\hline $\mathrm{VO}_{2}$ máx. $(\mathrm{ml} / \mathrm{kg} / \mathrm{min})$ & $36,0(26,0-48,0)$ & $47,0(41,0-58,0)$ & $0,008^{+*}$ \\
\hline Cigarros/dia & $20,0(20,0-32,5)$ & --- & \\
\hline Anos-Maço & $30,0(17,5-39,0)$ & --- & \\
\hline \multicolumn{4}{|l|}{ Fagerström } \\
\hline Moderado (\%) & 32 & --- & \\
\hline Elevado (\%) & 48 & --- & \\
\hline Muito elevado (\%) & 20 & --- & \\
\hline
\end{tabular}

n: número de indivíduos; F/M: feminino/masculino; kg: quilogramas; IMC: índice de massa corporal; VEF; volume expiratório forçado no primeiro segundo; CVF: capacidade vital forçada; DTC6: distância percorrida no teste de caminhada de seis minutos; $\mathrm{VO}_{2}$ máx: consumo máximo de oxigênio; $\mathrm{ml} / \mathrm{kg} / \mathrm{min}$ : mililitros por quilograma por minuto; Tabagista moderado: consumo de 15 a 24 cigarros por dia; Tabagista pesado: consumo $\geq 25$ cigarros por dia.

Dados expressos em mediana (intervalo interquartílico 25 e 75\%)

t: Teste T de Student para amostras não-pareadas

‡: Teste de Mann-Whitney

$\delta$ : Teste qui-quadrado

*Diferença estatística significante $(p<0,05)$

$\mathrm{Na}$ análise intragrupo tanto no exercício submáximo quanto máximo houve redução no TTS pós-exercício em ambos os grupos. Já nos índices da VFC o grupo tabagista apresentou redução significativa apenas do intervalo RR e aumento significativo da FC em comparação ao momento pré-exercício submáximo enquanto que no grupo controle foram observados valores significativamente menores dos índices RMSSD, intervalo RR, HFms ${ }^{2}$ e SD1 e aumento significativo da FC no pós-exercício em comparação ao momento pré-exercício submáximo. Com relação ao exercício máximo, ambos os grupos apresentaram redução significativa dos índices SDNN, RMSSD, intervalo RR, $\mathrm{LFms}^{2}, \mathrm{HFms}^{2}$ e un, SD1 e SD2 e aumento dos índices FC, LFun e relação LF/HF (Tabela 2).

Tabela 2. Dados de monoximetria, TMC e variabilidade da frequência cárdica dos grupos antes e após exercício submáximo e máximo

\begin{tabular}{|c|c|c|c|c|c|c|}
\hline \multirow{2}{*}{ Variáveis } & \multicolumn{3}{|c|}{ Grupo Tabagista $(n=25)$} & \multicolumn{3}{|c|}{ Grupo Controle $(n=15)$} \\
\hline & Repouso & Pós-exercício & $P$ & Repouso & Pós-exercício & $P$ \\
\hline \multicolumn{7}{|c|}{ Exercício Submáximo } \\
\hline COex (ppm) & $7,0(5,0-9,0)$ & $7,0(5,0-9,0)$ & $0,100^{+}$ & $2,0(1,0-2,0)$ & $1,0(0,0-2,0)$ & $0,075 \ddagger$ \\
\hline TTS(min.) & $14,7(9,1-18,4)$ & $7,3(5,9-9,6)$ & $0,002 t^{*}$ & $11,0(7,8-13,2)$ & $7,7(5,7-9,0)$ & $0,023 t^{*}$ \\
\hline FC (bpm) & $71,0(64,5-79,0)$ & $80,0(67,5-87,5)$ & $0,002^{+*}$ & $71,0(68,0-76,0)$ & $82,0(75,0-88,0)$ & $<0,0001^{+*}$ \\
\hline SDNN (ms) & $30,5(24,0-37,1)$ & $28,7(24,9-36,4)$ & $0,514 \ddagger$ & $29,4(21,4-32,2)$ & $20,3(15,6-29,2)$ & $0,083 \ddagger$ \\
\hline RMSSD (ms) & $25,2(17,8-29,7)$ & $21,0(17,2-29,3)$ & $0,268 \ddagger$ & $21,0(16,5-23,5)$ & $11,5(10,2-21,0)$ & $0,031^{+*}$ \\
\hline $\mathrm{RR}$ (ms) & $844,0(767,0-902,0)$ & $779,0(724,5-870,5)$ & $0,037^{+*}$ & $827,0(742,0-881,0)$ & $757,0(688,0-838,0)$ & $<0,0001 t^{*}$ \\
\hline $\operatorname{LF}\left(m s^{2}\right)$ & $509,0(326,0-840,0)$ & $569,0(248,5-897,5)$ & $0,619^{+}$ & $502,0(314,0-685,0)$ & $369,0(157,0-511,0)$ & $0,496^{+}$ \\
\hline $\mathrm{HF}\left(\mathrm{ms}^{2}\right)$ & $208,0(114,0-300,5)$ & $199,0(92,5-302,5)$ & $0,989^{+}$ & $159,0(85,0-270,0)$ & $104,0(38,0-156,0)$ & $0,029^{+*}$ \\
\hline LF (un) & $70,5(58,2-82,0)$ & $73,8(65,3-85,5)$ & $0,339^{+}$ & $73,9(61,4-82,3)$ & $82,2(66,9-86,1)$ & $0,105 \ddagger$ \\
\hline HF (un) & $24,8(18,0-41,6)$ & $27,6(15,7-37,7)$ & $0,469 \ddagger$ & $26,1(17,7-38,6)$ & $17,8(13,8-33,1)$ & $0,104 \ddagger$ \\
\hline $\mathrm{LF} / \mathrm{HF}\left(\mathrm{ms}^{2}\right)$ & $2,4(1,4-4,6)$ & $2,8(1,9-5,9)$ & $0,581^{+}$ & $2,8(1,6-4,7)$ & $4,6(2,0-6,2)$ & $0,179 \ddagger$ \\
\hline SD1 (ms) & $17,8(12,6-21,0)$ & $14,9(12,2-20,8)$ & $0,269 \ddagger$ & $14,8(11,7-16,6)$ & $8,2(7,2-14,9)$ & $0,031^{+*}$ \\
\hline $\mathrm{SD} 2(\mathrm{~ms})$ & $38,5(31,3-48,2)$ & $38,3(32,3-47,6)$ & $0,577 \ddagger$ & $37,4(28,0-42,8)$ & $27,8(21,2-38,8)$ & $0,106 \ddagger$ \\
\hline
\end{tabular}


Tabela 2. Continuação

\begin{tabular}{|c|c|c|c|c|c|c|}
\hline \multirow{2}{*}{ Variáveis } & \multicolumn{3}{|c|}{ Grupo Tabagista $(n=25)$} & \multicolumn{3}{|c|}{ Grupo Controle $(n=15)$} \\
\hline & Repouso & Pós-exercício & $P$ & Repouso & Pós-exercício & $P$ \\
\hline \multicolumn{7}{|c|}{ Exercício Máximo } \\
\hline Coex (ppm) & $7,0(4,5-11,0)$ & $6,0(4,0-10,0)$ & $0,075 \ddagger$ & $1,0(0,0-2,0)$ & $0,0(0,0-2,0)$ & $0,034 t^{*}$ \\
\hline TTS(min.) & $12,2(9,1-18,7)$ & $8,1(6,0-11,0)$ & $0,006 t^{*}$ & $8,7(6,9-13,7)$ & $5,9(5,0-7,5)$ & $0,017^{+*}$ \\
\hline $\mathrm{FC}(\mathrm{bpm})$ & $74,0(68,0-78,5)$ & $96,0(84,5-103,0)$ & $<0,0001^{+*}$ & $76,0(72,0-84,0)$ & $102,0(98,0-106,0)$ & $<0,0001^{+*}$ \\
\hline SDNN (ms) & $27,0(22,7-36,4)$ & $16,9(12,4-23,2)$ & $<0,00011^{*}$ & $31,0(24,5-34,7)$ & $13,5(10,7-18,4)$ & $<0,0001 t^{*}$ \\
\hline RMSSD (ms) & $24,1(18,0-29,6)$ & $10,1(6,0-16,1)$ & $<0,0001^{+*}$ & $20,5(15,5-23,3)$ & $6,6(6,0-9,9)$ & $0,001^{+*}$ \\
\hline $\mathrm{RR}(\mathrm{ms})$ & $820,0(762,5-887,0)$ & $663,0(589,5-725,0)$ & $<0,0001 t^{*}$ & $779,0(745,0-857,0)$ & $613,0(593,0-642,0)$ & $<0,0001 t^{*}$ \\
\hline $\operatorname{LF}\left(\mathrm{ms}^{2}\right)$ & $412,0(279,5-599,0)$ & $187,0(119,0-323,0)$ & $0,002^{+*}$ & $488,0(289,0-746,0)$ & $149,0(61,0-260,0)$ & $0,003^{+*}$ \\
\hline $\mathrm{HF}\left(\mathrm{ms}^{2}\right)$ & $159,0(114,0-319,0)$ & $31,0(12,0-108,0)$ & $<0,0001^{+*}$ & $198,0(136,0-277,0)$ & $14,0(8,0-40,0)$ & $0,001^{+*}$ \\
\hline LF (un) & $71,0(60,0-80,5)$ & $86,5(81,6-90,2)$ & $<0,0001^{+*}$ & $69,4(61,2-77,2)$ & $85,8(79,3-93,3)$ & $0,001^{+*}$ \\
\hline HF (un) & $29,0(19,5-40,0)$ & $13,5(9,7-18,3)$ & $<0,0001^{+*}$ & $30,6(22,8-38,8)$ & $14,2(6,7-20,7)$ & $0,001^{+*}$ \\
\hline $\mathrm{LF} / \mathrm{HF}\left(\mathrm{ms}^{2}\right)$ & $2,5(1,5-4,1)$ & $6,4(4,5-9,3)$ & $<0,0001^{+*}$ & $2,3(1,6-3,4)$ & $6,1(3,8-14,0)$ & $0,004 t^{*}$ \\
\hline $\mathrm{SD} 1(\mathrm{~ms})$ & $16,6(12,7-20,0)$ & $7,2(4,2-11,4)$ & $<0,0001^{+*}$ & $14,5(11,0-16,5)$ & $4,7(4,2-7,0)$ & $0,001^{+*}$ \\
\hline SD2 (ms) & $35,0(29,4-46,3)$ & $22,6(16,7-31,1)$ & $<0,0001 t^{*}$ & $41,3(31,9-44,8)$ & $18,5(14,7-25,3)$ & $<0,0001 t^{*}$ \\
\hline
\end{tabular}

COex: monóxido de carbono no ar exalado; ppm: partes por milhão; TTS: tempo de trânsito de sacarina; min: minutos; FC: frequência cardíaca; bpm: batimentos por minuto; SDNN: desvio-padrão da média de todos os intervalos RR normais, expresso em milissegundos; RMSSD: raiz quadrada da média do quadrado das diferenças entre intervalos RR normais adjacentes em um intervalo de tempo, expresso em milissegundos; LF: baixa frequência; HF: alta frequência; ms: milissegundos; nu: unidades normalizadas; SD1: desvio-padrão da variabilidade instantânea batimento-a-batimento; SD2: desvio-padrão da variabilidade a longo prazo.

Dados expressos em mediana (intervalo interquartílico 25 e 75\%)

t: Teste t de Student para amostra pareadas;

f: Teste de Wilcoxon;

*Diferença estatística significante $(p<0,05)$

A Tabela 3 apresenta as análises dos deltas intragrupos e entre os grupos. O grupo tabagista apresentou diminuição significativa durante o exercício máximo em comparação ao exercício submáximo para os índices SDNN, RMSSD, intervalo RR, LFms ${ }^{2}, \mathrm{HFms}^{2}$ e un,
SD1 e SD2 e aumento no índice FC e LF/HF. No grupo controle também houve diminuição significativa dos índices SDNN, RMSSD, intervalo RR, $\mathrm{HFms}^{2}$ e un, $\mathrm{SD} 1$ e SD2 e aumento dos índices FC, LFun e LF/HF no exercício máximo comparado com o submáximo.

Tabela 3. Magnitude de resposta dos grupos avaliados de acordo com os deltas de exercício submáximo e máximo na monoximetria, transporte mucociliar e índices de variabilidade da frequência cardíaca. Dados expressos em média e desvio-padrão

\begin{tabular}{|c|c|c|c|c|c|c|c|c|}
\hline & \multicolumn{3}{|c|}{ Exerc. Submáximo } & \multicolumn{3}{|c|}{ Exerc. Máximo } & \multirow{2}{*}{$\begin{array}{c}\text { Grupo Tabagista } \\
P(\text { Submáximo } \\
\text { vs. Máximo })\end{array}$} & \multirow{2}{*}{$\begin{array}{c}\text { Grupo Controle } \\
\text { P(Submáximo } \\
\text { vs. Máximo) }\end{array}$} \\
\hline & Grupo Tabagista & Grupo Controle & $P$ & Grupo Tabagista & Grupo Controle & $P$ & & \\
\hline COex (ppm) & $-0,6 \pm 1,6$ & $-0,7 \pm 1,4$ & $1^{+}$ & $-0,4 \pm 1,6$ & $-0,6 \pm 1,3$ & $0,244^{+}$ & $0,527 \delta$ & $0,154 \delta$ \\
\hline TTS (min) & $-5,8 \pm 8,7$ & $-2,1 \pm 3,5$ & $0,067 \ddagger$ & $-4,9 \pm 8,1$ & $-4,3 \pm 6,2$ & $0,806 \ddagger$ & $0,847 \S$ & $0,777 \S$ \\
\hline $\mathrm{FC}(\mathrm{bpm})$ & $6,3 \pm 9,1$ & $22,2 \pm 15,7$ & 0,303 & $9,1 \pm 6,6$ & $23,3 \pm 11,3$ & 0,813 & $<0,0001 \S^{*}$ & $<0,0001 \S^{*}$ \\
\hline SDNN (ms) & $-1,1 \pm 8,3$ & $-5,4 \pm 11,2$ & $0,173 \ddagger$ & $-11,2 \pm 8,8$ & $-14,3 \pm 9,8$ & $0,380 \ddagger$ & $<0,0001 \S^{*}$ & $0,033 \S^{*}$ \\
\hline RMSSD (ms) & $-1,5 \pm 6,7$ & $-5,0 \pm 8,0$ & $0,152 \ddagger$ & $-21,9 \pm 42,6$ & $-13,0 \pm 8,5$ & $0,600^{+}$ & $<0,0001 \delta^{*}$ & $0,008 \S^{*}$ \\
\hline $\mathrm{RR}(\mathrm{ms})$ & $-28,8 \pm 70,1$ & $-56,6 \pm 47,7$ & $0,125^{+}$ & $-156,9 \pm 90,0$ & $-169,0 \pm 39,5$ & $0,563 \ddagger$ & $<0,0001 \delta^{*}$ & $<0,0001 \S^{*}$ \\
\hline $\operatorname{LF}\left(m s^{2}\right)$ & $27,4 \pm 409,8$ & $-80,0 \pm 440,8$ & $0,440 \ddagger$ & $-251,6 \pm 382,4$ & $-309,5 \pm 308,2$ & $0,543^{+}$ & $0,026 \delta^{*}$ & $0,124 \S$ \\
\hline $\mathrm{HF}\left(\mathrm{ms}^{2}\right)$ & $-14,7 \pm 162,5$ & $-85,6 \pm 151,0$ & $0,106^{+}$ & $-152,2 \pm 175,5$ & $-187,6 \pm 154,7$ & $0,507^{+}$ & $<0,006 \delta^{*}$ & $0,027 \delta^{*}$ \\
\hline LF (un) & $23,0 \pm 98,5$ & $7,4 \pm 16,6$ & $0,581^{+}$ & $15,5 \pm 12,8$ & $16,4 \pm 8,9$ & $0,790 \ddagger$ & $0,104 \delta$ & $0,033 \S^{*}$ \\
\hline HF (un) & $-2,9 \pm 19,6$ & $-7,4 \pm 16,6$ & $0,458 \ddagger$ & $-15,4 \pm 12,7$ & $-16,4 \pm 8,8$ & $0,796 \neq$ & $0,018 \S^{*}$ & $0,033 \S^{*}$ \\
\hline $\mathrm{LF} / \mathrm{HF}\left(\mathrm{ms}^{2}\right)$ & $2,5 \pm 11,7$ & $1,2 \pm 3,3$ & $0,489^{+}$ & $5,7 \pm 6,8$ & $8,9 \pm 10,1$ & $0,292^{+}$ & $0,014 \delta^{*}$ & $0,004 \delta^{*}$ \\
\hline $\mathrm{SD} 1(\mathrm{~ms})$ & $-1,1 \pm 4,8$ & $-3,5 \pm 5,7$ & $0,154 \ddagger$ & $-9,7 \pm 6,2$ & $-9,2 \pm 6,0$ & $0,800 \ddagger$ & $<0,0001 \S^{*}$ & $0,008 \S^{*}$ \\
\hline SD2 (ms) & $-1,3 \pm 11,2$ & $-6,7 \pm 15,1$ & $0,198 \ddagger$ & $-13,0 \pm 11,6$ & $-18,1 \pm 12,8$ & $0,202 \ddagger$ & $<0,0001 \S^{*}$ & $0,040 \S^{*}$ \\
\hline
\end{tabular}

COex: monóxido de carbono no ar exalado; ppm: partes por milhão; TTS: tempo de trânsito de sacarina; min: minutos; FC: frequência cardíaca; bpm: batimentos por minuto; SDNN: desvio-padrão da média de todos os intervalos RR normais, expresso em milissegundos; RMSSD: raiz quadrada da média do quadrado das diferenças entre intervalos RR normais adjacentes em um intervalo de tempo, expresso em milissegundos; LF: baixa frequência; HF: alta frequência; ms: milissegundos; nu: unidades normalizadas; SD1: desvio-padrão da variabilidade instantânea batimento-a-batimento; SD2: desvio-padrão da variabilidade a longo prazo

t: Teste de Mann Whitney

‡: Teste T de Student para amostras não pareadas

$\delta$ : Teste de Wilcoxon

$\S$ : Teste T de Student para amostras pareadas

*Diferença estatística significante $(p<0,05)$. 
Houve correlação positiva entre TTS e o índice LFms ${ }^{2}$ $(\mathrm{r}=0,520 ; \mathrm{p}=0,008)$ no exercício máximo para o grupo tabagista e correlação negativa entre TTS e os índices SDNN ( $r=-0,833 ; p<0,0001)$, RMSSD ( $r=-0,614$; $\mathrm{p}=015)$, HFms2 ( $r=-0,645 ; \mathrm{p}=0,009), \mathrm{SD} 1(\mathrm{r}=-0,623$, $\mathrm{p}=0,013)$ e SD2 $(\mathrm{r}=-0,832 ; \mathrm{p}<0,0001)$ no grupo controle pós exercício máximo.

\section{DISCUSSÃO}

Os resultados do presente estudo mostraram aceleração do TMC nasal de tabagistas e não tabagistas após uma sessão de exercício físico submáximo e máximo. Com relação à VFC, no exercício submáximo ambos os grupos apresentaram redução do intervalo RR e aumento da FC e no grupo não tabagista também foram observadas reduções dos índices RMSSD, $\mathrm{HFms}^{2}$ e SD1 e aumento dos índices LFun e relação LF/HF, no entanto não houve diferença na magnitude de resposta entre os grupos neste estímulo. Já para o exercício máximo ambos os grupos apresentaram alterações nos índices da VFC caracterizadas por diminuição da modulação parassimpática e aumento dos índices FC, LFun e relação LF/HF. Além disso, foi observado uma correlação positiva entre o TTS e o índice $\mathrm{LFms}^{2}$ no grupo tabagista e correlação negativa entre TTS e os índices SDNN, RMSSD, HFms², SD1 e SD2 no grupo não tabagista pós exercício máximo.

A aceleração do TMC em ambos os grupos após o exercício corrobora com resultados obtidos em outros estudos ${ }^{10,11,24,30}$. Ramos et al. ${ }^{24}$ compararam o comportamento do TMC de indivíduos tabagistas frente a três estímulos (exercício aeróbico moderado isolado 60-70\% $\mathrm{VO}_{2}$ max, exercício aeróbico moderado combinado ao fumo e fumo isolado) e verificaram que todos os estímulos aceleraram o TMC em comparação ao repouso. Outros autores também encontraram aceleração do TMC frente ao exercício, porém em indivíduos saudáveis ${ }^{10,11}$.

A resposta mucociliar acelerada frente ao exercício físico parece ser influenciada, pelo menos em parte, pelo sistema nervoso autônomo (SNA), pois a hiperventilação pulmonar estimula os receptores centrais (quimioceptores) que por sua vez, estimulam a atividade autonômica do ramo simpático e como consequência, há o aumento dos níveis de catecolaminas (epinefrina e norepinefrina) que aceleram o batimento ciliar ${ }^{10,31}$. Concomitantemente, essa hiperventilação promove a irradiação cortical sobre a região bulbar que resulta na retirada vagal progressiva ${ }^{32}$, potencializando a atuação simpática sobre o batimento ciliar e reduzindo o TTS $^{10}$.

Após o exercício submáximo e máximo, tanto tabagistas como não tabagistas apresentaram redução dos intervalos $\mathrm{RR}$ (ms) e aumento da FC, indicando que a frequência cardíaca desses indivíduos se mantém elevada no período pós-exercício analisado. No exercício submáximo apenas o grupo controle apresentou diminuição significativa dos índices RMSSD, $\mathrm{HFms}^{2}$ e SD1 no período pós exercício em comparação ao repouso, sugerindo que esses indivíduos ainda não tiveram recuperação da modulação parassimpática pós-exercício. Esse comportamento não foi observado no grupo tabagista que, exceto para os intervalos $\mathrm{RR}$, não apresentou diferenças significativas para os índices de VFC nessa mesma condição, o que indica que esses indivíduos estavam recuperados do ponto de vista autonômico. Os resultados apresentados podem estar relacionados à menor intensidade realizada no TC6' pelos tabagistas, pois neste teste o participante faz seu próprio ritmo, diferente do teste máximo onde se é imposto uma velocidade e inclinação até o seu esforço máximo. Deste modo, a recuperação da modulação autonômica cardíaca no trabalho submáximo com menor intensidade é mais rápida, o que pode ter acontecido antes do término da avaliação do TMC nasal neste grupo.

Já no exercício máximo, ambos os grupos apresentaram respostas autonômicas que indicam que eles ainda não se recuperaram no momento avaliado. Durante exercícios de alta intensidade a recuperação é mais lenta, pois as alterações induzidas no exercício são mais intensas ${ }^{33,34} \mathrm{O}$ que justifica as respostas apresentadas e permite avaliar a influência dessas modificações no TMC nasal.

Em relação à correlação da modulação autonômica e TMC nasal, os resultados mostraram uma correlação positiva entre oTTS e o índice LFms ${ }^{2}$ no grupo tabagista e correlação negativa entre TTS e os índices SDNN, $\mathrm{RMSSD}, \mathrm{HFm}{ }^{2}$, SD1 e SD2 no grupo não tabagista pós exercício máximo. Tais alterações demonstram a influência dos ramos simpático e parassimpático do SNA sobre o batimento ciliar após o exercício máximo em ambos os grupos. Essa influência não foi observada com o exercício submáximo, pois este tipo de exercício produz menores efeitos sobre a modulação autonômica, sendo assim o aumento do TTS pode ter sido em partes provocado por efeitos mecânicos da hiperventilação pulmonar ${ }^{14}$. Resultado semelhante a esse, foi também encontrado por Santos et al. ${ }^{30}$ que também não observaram nenhuma associação entre a modulação autonômica e a função mucociliar de tabagistas. 
Como limitação do estudo podemos salientar a falta da avaliação do TMC em diferentes momentos pósexercício, o que poderia auxiliar na melhor compreensão da ação da modulação autonômica e hiperventilação pulmonar sobre o batimento ciliar nasal. Outra limitação que pode ser considerada foi a ausência de um eletrocardiograma que pudesse assegurar que os indivíduos avaliados não apresentassem arritmias. No entanto, todos os cuidados necessários foram tomados em relação à captação da frequência cardíaca que originou a série de intervalos RR, exclusão de séries com mais de $5 \%$ de erro e filtragem e inspeção visual da série, o que nos deu segurança para sua utilização e, mesmo que o indivíduos apresentasse algum distúrbio de ritmo, isso não influenciou os resultados.

O presente estudo tem importante relevância clínica, pois sugere que a intensidade do exercício aeróbico influencia a resposta do TMC nasal de indivíduos tabagistas e não tabagistas aumentando a eficácia da função mucociliar no período pós-exercício, o que reflete em um mecanismo de defesa pulmonar mais eficiente contra infecções respiratórias e agentes nocivos ao trato respiratório. Além disso, os resultados mostraram também que a intensidade do exercício está relacionada a participação do SNA nesse processo.

\section{CONCLUSÃO}

Conclui-se que independente da intensidade do exercício aeróbico ocorre aumento do TMC nasal em tabagistas, no entanto tal alteração parece ser influenciado pelo sistema autonômico apenas frente um exercício máximo.

\section{AGRADECIMENTOS}

Os autores agradecem a Fundação de Amparo à Pesquisa do Estado de São Paulo (FAPESP) pelo apoio financeiro no processo $n^{\circ}$ 2013/04091-0 que permitiu a realização deste estudo.

\section{REFERÊNCIAS}

1. Santos APS, Ramos D, Oliveira GM, Santos AAS, Freire APCF, Ito JT, et al. Influence of smoking consumption and nicotine dependence degree in cardiac autonomic modulation. Arq Bras Cardiol. 2016;106(6):510-18. doi: 10.5935/abc.20160063
2. Proença MGL, Xavier RF, Ramos D, Cavalheri V, Ramos EMC. Immediate and short term effects of smoking on nasal mucociliary clearance in smokers. Rev Port Pneumol. 2011;17(4):172-6. doi: 10.1016/j.rppneu.2010.12.001

3. Xavier RF, Ramos D, Ito JT, Rodrigues FMM, Bertolini GN, Macchione $M$, et al. Effects of cigarette smoking intensity on the mucociliary clearance of active smokers. Respiration. 2013;86(6):479-85. doi: 10.1159/000348398

4. Manzano BM, Vanderlei LCM, Ramos EM, Ramos D. Efeitos agudos do tabagismo sobre a modulação autonômica: análise por meio do plot de Poincaré. Arq Bras Cardiol. 2011;96(2):154-60. doi: 10.1590/S0066-782X2011005000013

5. Leopold PL, O'Mahony MJ, Lian XJ, Tilley AE, Harvey BG, Cristal RG. Smoking is associated with shortened airway cilia. PLoS One. 2009;4(12):e8157. doi: 10.1371/journal.pone.0008157

6. Santos UP. Relevância da anamnese e de biomarcadores na avaliação do tabagismo entre os pacientes com doença das vias aéreas. J Bras Pneumol. 2015;41(2):105-6. doi: 10.1590/ S1806-37132015000200001

7. Ramos EMC, Toledo AC, Xavier RF, Fosco LC, Vieira, RP, Ramos D, et al. Reversibility of impaired nasal mucociliary clearance in smokers following a smoking cessation programme. Respirology. 2011;16(5):849-55. doi: 10.1111/j.1440-1843.2011.01985.x

8. Proença M, Pitta F, Kovelis D, Mantoani LC, Furlanetto KC, Zabatiero J, et al. Mucociliary clearance and its relation with the level of physical activity in daily life in healthy smokers and nonsmokers. Rev Port Pneumol. 2012;18(5):233-8. doi: 10.1016/j.rppneu.2012.03.003

9. Droguett VSL, Santos AC, Medeiros CE, Marques DP, Nascimento LS, Brasileiro-Santos MS. Cardiac autonomic modulation in healthy elderly after different intensities of dynamic exercise. Clin Interv Aging. 2015;10:203-8. doi: 10.2147/CIA.S62346

10. Woff RK, Dolovick MB, Obminsk G, Newhouse MT. Effects of exercise and eucapnic hyperventilation on bronchial clearance in man. J Appl Physiol Respir Environ Exerc Physiol. 1977;43(1):46-50. doi: 10.1152/jappl.1977.43.1.46

11. Saketkhoo K, Kaplan I, Sackner MA. Effect of exercise on nasal mucous velocity and nasal airflow resistance in normal subjects. J Appl Physiol Respir Environ Exerc Physiol. 1979;46(2):369-71. doi: 10.1152/jappl.1979.46.2.369

12. Meneses-Gaya IC, Zuardi AW, Loureiro SR, Crippa JAS. As propriedades psicométricas do teste de fagerström para dependência de nicotina. J Bras Pneumol. 2009;35(1):73-82. doi: 10.1590/S1806-37132009000100011

13. SRNT Subcommittee on Biochemical Verification. Biochemical verification of tobacco use and cessation. Nicotine Tob Res. 2002;4(2):149-59. doi: 10.1080/14622200210123581

14. Chatkin J, Fritscher L, Abreu C, Cavalet-Blanco D, Chatkin G, Wagner $\mathrm{M}$, et al. Exhaled carbon monoxide as a marker for evaluating smoking abstinence in a Brazilian population sample. Prim Care Respir J. 2007;16(1):36-40. doi: 10.3132/pcrj.2007.00008

15. Miller MR, Hankinson J, Brusasco V, Burgos F, Casaburi R, Coates A, et al. Standardization of spirometry. Eur Respir J. 2005;26(2):319-38. doi: 10.1183/09031936.05.00034805

16. Duarte AA, Pereira CAC, Rodrigues SC. Validation of new Brazilian predicted values for forced spirometry in Caucasians and comparison with predicted values obtained using other 
reference equations. J Bras Pneumol. 2007;33(5):527-35. doi: 10.1590/S1806-37132007000500007

17. American Thoracic Society. ATS statement: guidelines for the six-minute walk test. Am J Respir Crit Care Med. 2002;166(1):111-7. doi: 10.1164/ajrccm.166.1.at1102

18. Bentley DJ, Newell J, Bishop D. Incremental exercise test design and analysis: implications for performance diagnostics in endurance athletes. Sports Med. 2007;37(7):575-86. doi: 10.2165/00007256-200737070-00002

19. Esteve-Lanao J, Foster C, Seiler S, Lucia A. Impact of training intensity distribution on performance in endurance athletes. J Strength Cond Res. 2007;21(3):943-9. doi: 10.1519/R-19725.1

20. Silva DF, Sotero RC, Simões HG, Machado FA. Máxima velocidade aeróbia calculada pelo custo da frequência cardíaca: relação com a performance. Rev Andal Med Deport. 2015;8(1):7-15. doi: 10.1016/j.ramd.2014.06.001

21. Silva GSF, Deresz CS, Lima PRJ. Associação entre limiares ventilatórios e percepção do esforço. Rev Bras Cienc Mov [Internet]. 2006 [cited 2017 Nov 15];14(2):79-86. Availale from: https://portalrevistas.ucb.br/index.php/RBCM/article/ viewFile/690/695

22. Vanderlei LCM, Silva RA, Pastre CM, Azevedo FM, Godoy MF. Comparison of the Polar S810i monitor and the ECG for the analysis of heart rate variability in the time and frequency domains. Braz J Med Biol Res. 2008;41(10):854-9. doi: 10.1590/S0100-879X2008005000039

23. Gamelin FX, Berthoin S, Bosquet L. Validity of the polar $\$ 810$ heart rate monitor to measure R-R intervals at rest. Med Sci Sports Exerc. 2006;38(5):887-93. doi: 10.1249/01.mss.0000218135.79476.9c

24. Ramos EM, Vanderlei LC, Ito JT, Lima FF, Rodrigues FM, Manzano BM, et al. Acute mucociliary clearance response to aerobic exercise in smokers. Respir Care. 2015;60(11):1575-84. doi: 10.4187/respcare.04093

25. Stanley P, MacWilliam L, Greenstone M, Mackay I, Cole P. Efficacy of saccharin test for screening to detect abnormal mucociliary clearance. $\mathrm{Br} J$ Dis Chest. 1984;78:62-5. doi: 10.1016/0007-0971(84)90098-6

26. Tarvainen MP, Niskanen JP, Lipponen JA, Ranta-Aho PO, Karjalainen PA. Kubios HRV: heart rate variability analysis software. Comput Methods Programs Biomed. 2014;113(1):210-20. doi: 10.1016/j.cmpb.2013.07.024

27. Vanderlei LCM, Pastre CM, Hoshi RA, Carvalho TD, Godoy MF. Noções básicas de variabilidade da frequência cardíaca e sua aplicabilidade clínica. Rev Bras Cir Cardiovasc. 2009;24(2):205-17. doi: 10.1590/S0102-76382009000200018

28. Ferreira MT, Messias M, Vanderlei LCM, Pastre CM. Caracterização do comportamento caótico da variabilidade da frequência cardíaca (VFC) em jovens saudáveis. Tema. 2010;11(2):141-50. doi: 10.5540/tema.2010.011.02.0141

29. Godoy MF, Takakura IT, Correa PR. Relevância da análise do comportamento dinâmico não linear (Teoria do Caos) como elemento prognóstico de morbidade e mortalidade em pacientes submetidos à cirurgia de revascularização miocárdica. Arq Cienc Saude [Internet]. 2005 [cited 2017 Nov 15];12(4):167-71. Available from: http://repositorio-racs.famerp.br/racs_ol/ vol-12-4/01_ID175.pdf

30. Santos APS, Ramos D, Ito JT, Toledo AC, Vanderlei LCM, Ramos EMC. Efeito do esforço físico submáximo na modulação autonômica cardíaca e no transporte mucociliar de tabagistas. Rev Inspirar [Internet]. 2012 [cited 2018 Feb 10];4(5):20-1. Available from: https://www.inspirar.com.br/wp-content/ uploads/2016/05/suplemento-marilia-set-out-2012-1.pdf

31. Kraemer WJ, Gordon SE, Fragala MS, Bush JA, Szivak TK, Flanagan SD, et al. The effects of exercise training programs on plasma concentrations of proenkephalin peptide $\mathrm{F}$ and catecholamines. Peptides. 2015;64:74-81. doi: 10.1016/ j.peptides.2015.01.001

32. Gallo L Jr, Maciel BC, Marin Neto JA, Martins LE. Sympathetic and parasympathetic changes in heart rate control during dynamic exercise induced by endurance training in man. Braz J Med Biol Res [Internet]. 1989 [cited 2018 Feb 10];22:631-43. Available from: https://www.ncbi.nlm.nih.gov/pubmed/2620172

33. Fadel PJ. Arterial baroreflex control of the peripheral vasculature in humans: rest and exercise. Med Sci Sports Exerc. 2008;40(12):2055-62. doi: 10.1249/MSS.0b013e318180bc80

34. Michael S, Jay O, Halaki M, Graham K, Davis GM. Submaximal exercise intensity modulates acute post-exercise heart rate variability. Eur J Appl Physiol. 2016;116(4):697-706. doi: 10.1007/s00421-016-3327-9 